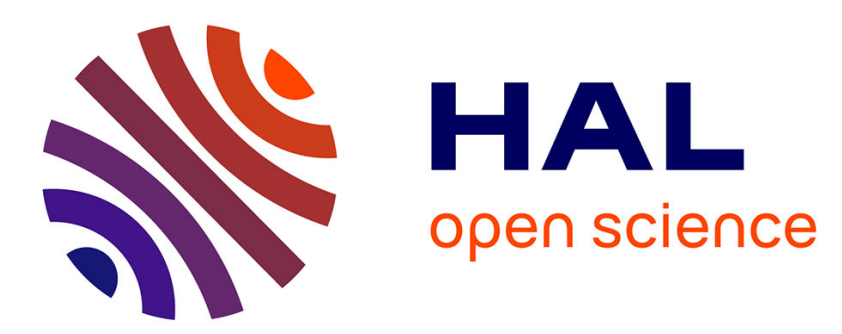

\title{
Optimizing the anaerobic digestion of microalgae in a coupled process
}

\author{
Térence Bayen, Francis Mairet, Pierre Martinon, Matthieu Sebbah
}

\section{To cite this version:}

Térence Bayen, Francis Mairet, Pierre Martinon, Matthieu Sebbah. Optimizing the anaerobic digestion of microalgae in a coupled process. 13th European Control Conference, Jul 2013, Zurich, Switzerland. pp.6. hal-00754971

\section{HAL Id: hal-00754971 \\ https://hal.inria.fr/hal-00754971}

Submitted on 13 Feb 2013

HAL is a multi-disciplinary open access archive for the deposit and dissemination of scientific research documents, whether they are published or not. The documents may come from teaching and research institutions in France or abroad, or from public or private research centers.
L'archive ouverte pluridisciplinaire HAL, est destinée au dépôt et à la diffusion de documents scientifiques de niveau recherche, publiés ou non, émanant des établissements d'enseignement et de recherche français ou étrangers, des laboratoires publics ou privés. 


\title{
Optimizing the anaerobic digestion of microalgae in a coupled process
}

\author{
Terence Bayen ${ }^{1,4}$ and Francis Mairet ${ }^{2}$ and Pierre Martinon ${ }^{3}$ and Matthieu Sebbah ${ }^{4}$
}

\begin{abstract}
This work is devoted to maximizing the production of methane in a bioreactor coupling an anaerobic digester and a culture of micro-algae limited by light. The decision parameter is the dilution rate which is chosen as a control, and we enforce periodic constraints in order to repeat the same operation every day. The system is gathered into a three-dimensional system taking into account a day-night model of the light in the culture of micro-algae. Applying Pontryagin maximum principle, the necessary conditions on optimal trajectories indicate that the control consists of bang and/or singular arcs. We provide numerical simulations by both direct and indirect methods, which show the link between the light model and the structure of optimal solutions.
\end{abstract}

\section{INTRODUCTION}

Microalgae are cultivated nowadays for feed, food, or cosmetics [8], and have recently emerged as an attractive alternative for sustainable energy production [2]. Anaerobic digestion can be applied to convert microalgae biomass into biogas [4]. This process not only recovers the energy stored in biomass, but also leads to ammonium and phosphate release, which can in turn be source of nutrients for the microalgae culture. Coupling microalgae culture and anaerobic digestion is therefore a promising process to convert solar energy into methane. However, due to their inherent complexity, the control and optimization of such coupled systems present many challenges.

The model we consider combines a first reactor in which microalgae are cultivated and a second one where the microalgae are converted into biogas. Our aim is to find an optimal feeding strategy in order to maximize the production of biogas in the second reactor during one day. We impose periodic constraints on the system in order to repeat the same operation during a large number of periods. We follow [1] to describe the evolution of the concentration of microalgae limited by light in the first reactor. In the second reactor we use a model of chemostat (see [11]) to describe the evolution of gas converted by the biomass. In addition to the discontinous light model in [1], we also investigate smoother models for the day-night transition.

\footnotetext{
${ }^{1}$ Université Montpellier 2, Case courrier 051, 34095 Montpellier cedex 5, France. tbayen@math. univ-montp2. fr

${ }^{2}$ Departamento de Matemática, Universidad Técnica Federico Santa María, Avda. España 1680, Valparaíso, Chile. francis.mairet@usm.cl

${ }^{3}$ INRIA Saclay, Ecole Polytechnique, CMAP 91128 Palaiseau, France. pierre.martinondinria.fr

'INRA-INRIA 'MODEMIC' team, INRIA Sophia-Antipolis Méditerranée, UMR INRA-SupAgro 729 'MISTEA' 2 place Viala 34060 Montpellier. msebbah@inria.fr
}

The paper is organized in 5 sections. Section 2 presents the model for the coupled reactors. In section 3, we apply Pontraygin's Maximum Principle in order to derive necessary conditions on optimal trajectories. The optimal control consists in bang and/or singular arcs (see appendix for the exact expression of singular controls). Finally, section 4 is devoted to a numerical study of the problem, using both direct and indirect methods with the softwares Bocop and Shoot (see [5]). The direct method is used first to determine the optimal control structure, and also provides an initial guess for the indirect shooting.

In particular, we discuss the structure of the solution depending on the smoothness of the light model. In the discontinuous case, we find out that a singular arc cannot cross the day-night discontinuity. Then for a continuous (but not $C^{1}$ ) light model, the singular arc is admissible but the singular control is discontinuous. Finally, for a $C^{1}$ light model, the singular arc is admissible and the singular control is continuous.

\section{Statement of The PRoblem}

\section{A. Presentation of the model}

We consider that microalgae growth in the first reactor is limited by light. Therefore, we can use a similar modeling framework as the one presented in [1]: we assume that the specific growth rate is a decreasing function of microalgae (because of self-shading) and that the respiration rate $\rho$ is constant. The microalgae reactor of volume $V_{1}$ is fed with an input flow $Q_{1}$ taking values in $\left[0, Q_{1}^{\max }\right]$. Given some model simplifications (see [1]), the dynamics of microalgae $y$ writes:

$$
\frac{d y}{d t}=\frac{\nu(t) y}{\kappa+y}-\frac{Q_{1}}{V_{1}} y-\rho y
$$

where $\kappa>0$, and $\nu(t)$ represents the periodic light forcing. The output flow of the microalgae culture is sent in the anaerobic digester. The anaerobic digestion is represented by a classical bioreactor model (see e.g. [3]) and the substrate (i.e. the microalgae) is degradated by the biomass:

$$
\left\{\begin{array}{l}
\frac{d s}{d t}=-k \mu_{2}(s) x+\frac{Q_{2}}{V_{2}}(\alpha y-s), \\
\frac{d x}{d t}=\left[\mu_{2}(s)-\frac{Q_{2}}{V_{2}}\right] x,
\end{array}\right.
$$

where $\alpha>0, k>0$ are respectively an adimensionned concentration coefficient and a yield coefficient, and $x(t)$ is the concentration of biomass, $s(t)$ the concentration of substrate, $Q_{2}=Q_{1} / \alpha$ the input flow in the second reactor, $V_{2}$ the volume of this reactor, and $\mu_{2}$ the growth function in 
the second reactor. Gathering the two processes yields to the system:

$$
\left\{\begin{array}{l}
\frac{d y}{d t}=\frac{\nu(t) y}{\kappa+y}-\frac{Q_{1}}{V_{1}} y-\rho y, \\
\frac{d s}{d t}=-k \mu_{2}(s) x+\frac{Q_{2}}{V_{2}}(\alpha y-s), \\
\frac{d x}{d t}=\left[\mu_{2}(s)-\frac{Q_{2}}{V_{2}}\right] x .
\end{array}\right.
$$

For convenience, we perform the following change of variable: $(t, y, s, x) \leftarrow\left(\frac{Q_{1}^{\max }}{V_{1}} t, \frac{y}{\kappa}, \frac{s}{k}, x\right)$, and we set $u:=\frac{Q_{1}}{Q_{1}^{\max }}$. The system becomes:

$$
\left\{\begin{array}{l}
\frac{d y}{d t}=\frac{\mu(t) y}{1+y}-r y-u y \\
\frac{d s}{d t}=u \beta(\gamma y-s)-\mu_{2}(s) x \\
\frac{d x}{d t}=\left[\mu_{2}(s)-u \beta\right] x
\end{array}\right.
$$

with $r=\frac{\rho V_{1}}{Q_{1}^{\text {max }}}, \beta=\frac{V_{1}}{\alpha V_{2}}, \gamma=\frac{\alpha \kappa}{k}$. We assume that $\mu_{2}$ is of type Monod (see [11]), that is:

$$
\mu_{2}(s)=\mu_{2}^{m} \frac{s}{K_{s}+s},
$$

where $K_{s}>0$ and $\mu_{2}^{m}>0$ are given coefficients. We assume that for all $s$, we have

$$
\mu_{2}(s)<\beta
$$

This assumption is standard in order to control the concentration of $x$ in the reactor (see e.g. [6] in the case of a fed-batch reactor). The periodic forcing light $\nu$ is changed into $\mu$ (by the transformation above) with

$$
\mu(t)= \begin{cases}\bar{\mu}, & t \in[0, \bar{T}] \\ 0, & t \in(\bar{T}, T)\end{cases}
$$

Typically, we can take for $T=10$ one day, $\bar{T}=\frac{T}{2}$ and $\bar{\mu}>0$ is a given parameter. The values of all parameters used in the numerical section are given in table II-A.

TABLE I

VALUE OF THE PARAMETERS FOR (4)

\begin{tabular}{|l|l|l|}
\hline $\bar{\mu}=0.5$ & $r=0.005$ & $\gamma=1$ \\
$\mu_{2}^{m}=0.1$ & $K_{s}=0.05$ & $\beta=1$ \\
\hline
\end{tabular}

The next Lemma shows that system (4) satisfies an invariance property.

Lemma 1: The set $E=\mathbb{R}_{+}^{*} \times \mathbb{R}_{+}^{*} \times \mathbb{R}_{+}^{*}$ is invariant by the dynamics (4).

The proof uses standard arguments from differential equations such as Gronwall's Lemma. In the rest of the paper, we consider only initial conditions in $E$ for system (4).

\section{B. Optimization problem}

Our aim is to maximize the production of biogas in the second reactor (which is proportional to the biomass growth) with respect to the control $u(\cdot)$ under the constraint that the solution of (4) associated to $u(\cdot)$ is periodic, that is:

$$
y(0)=y(T), \quad s(0)=s(T), \quad x(0)=x(T) .
$$

Given this constraint, maximizing biomass growth $\mu_{2}(t) x(t)$ over a period is equivalent to maximizing $u(t) x(t)$, so the optimization problem reads as follows:

$$
\max _{u \in \mathcal{U}} J(u):=\int_{0}^{T} u(t) x_{u}(t) d t, \text { s.t. (8) holds, }
$$

where

$$
\mathcal{U}:=\{u: \mathbb{R} \rightarrow[0,1] \text { s.t. } u(\cdot) \text { meas., } T-\text { periodic }\},
$$

is the set of admissible controls, and $x_{u}$ is solution of (4). The existence of $T$-periodic trajectories for (4) can be proved by similar arguments as in [14].

Remark 1: From an environmental or economic point of view, the cost function to maximize is $\tilde{J}(u):=\frac{\beta}{\beta+c} J(u)$ where $c$ is a (environmental or economic) cost ratio of the two processes. Thus, $\beta:=\frac{V_{1}}{\alpha V_{2}}$ is a plant design parameter which should be chosen adequately in order to optimize the coupled process.

\section{NECESSARY CONDITIONS ON OPTIMAL TRAJECTORIES}

In this section, we apply Pontryagin maximum principle to derive necessary conditions on optimal trajectories for problem (9). The existence of an optimal control for (9) is standard by compactness arguments and the existence of $T$ periodic trajectories. We can then apply Fillipov's existence Theorem (see [7]).

\section{A. Pontryagin maximum principle}

The Hamiltonian associated to (9) is defined by $H=$ $H\left(y, s, x, \lambda_{1}, \lambda_{2}, \lambda_{3}, \lambda_{0}, u\right)$ :

$$
\begin{aligned}
H=\lambda_{1} & \left(\mu \frac{y}{1+y}-r y\right)+\left(\lambda_{3}-\lambda_{2}\right) \mu_{2}(s) x \\
& +u\left(-\lambda_{1} y+\lambda_{2} \beta(\gamma y-s)-\lambda_{3} \beta x+\lambda_{0} x\right),
\end{aligned}
$$

where $\lambda:=\left(\lambda_{1}, \lambda_{2}, \lambda_{3}\right)$ is the adjoint vector. Now, let $u$ an optimal control and $z:=(y(\cdot), x(\cdot), s(\cdot))$ the associated $T$ periodic trajectory. There exists $\lambda_{0} \leq 0$ and $\lambda:[0, T] \rightarrow \mathbb{R}^{3}$ such that the following adjoint equations are satisfied:

$$
\left\{\begin{array}{l}
\frac{d \lambda_{1}}{d t}=\left(-\frac{\mu}{(1+y)^{2}}+r+u\right) \lambda_{1}-u \lambda_{2} \beta \gamma \\
\frac{d \lambda_{2}}{d t}=x\left(\lambda_{2}-\lambda_{3}\right) \mu_{2}^{\prime}(s)+u \lambda_{2} \beta \\
\frac{d \lambda_{3}}{d t}=\left(\lambda_{2}-\lambda_{3}\right) \mu_{2}(s)+u \lambda_{3} \beta+\lambda_{0} u .
\end{array}\right.
$$

As the state is $T$-periodic, the adjoint vector $\lambda$ satisfies the transversality condition:

$$
\lambda(0)=\lambda(T),
$$

and is also $T$-periodic. Finally, we have the maximization condition: for a.e. $t \in[0, T], u(t)$ maximizes the function

$$
v \longmapsto H\left(t, z(t), \lambda(t), \lambda_{0}, v\right) \text {. }
$$

We assume that the optimal trajectory is a normal extremal, that is $\lambda_{0} \neq 0$ (by homogeneity, we take $\lambda_{0}=-1$ ). The switching function associated to the control is

$$
\psi:=-\lambda_{1} y+\lambda_{2} \beta(\gamma y-s)-\lambda_{3} \beta x+x,
$$


and it is $T$-periodic. From (13) an optimal control satisfies the following control law:

$$
\left\{\begin{array}{l}
u=1 \Longleftrightarrow \psi>0, \\
u=0 \Longleftrightarrow \psi<0,
\end{array}\right.
$$

When $u=1$ (resp. $u=0$ ) on some time interval, we say that the trajectory has an arc Bang + or $B_{+}$for short (resp. Bang or $\mathbf{B}_{-}$for short). If $\psi$ is zero on some time interval, we say that the trajectory has a singular arc. We call switching point a time $t_{0}$ at which the control is non-constant in any neighborhood of $t_{0}$.

From (4) and (6), we can see that the constant controls $u=0$ and $u=1$ are not solution of the problem (otherwise $x$ would be either increasing or decreasing on $[0, T]$ in contradiction with the periodicity). It follows that the trajectory necessarily contains a switching point (from an $\operatorname{arc}$ bang $_{ \pm}$to an $\operatorname{arc~bang}_{\mp}$ or to a singular arc). By the periodicity of the switching function, we have also the following property.

- If $\psi(0)>0$, then the control satisfies $u=0$ on $\left[0, t_{0}\right] \cup$ $\left[t_{1}, T\right]$ where $0<t_{0}<t_{1}<T$. Moreover, the trajectory contains at least two switching points on $[0, T]$.

- If $\psi(0)<0$, then the control satisfies $u=1$ on $\left[0, t_{0}^{\prime}\right] \cup$ $\left[t_{1}^{\prime}, T\right]$ where $0<t_{0}^{\prime}<t_{1}^{\prime}<T$. Moreover, the trajectory contains at least two switching points on $[0, T]$.

In other words, the periodic assumptions on the state-adjont system imply that the trajectory has the same structure in a neigborhood of $t=0$ and of $t=T$ in the case where $\psi(0)>0$ or $\psi(0)<0$.

\section{B. Computation of the singular arcs}

Let $\left[t_{1}, t_{2}\right]$ be a time interval where the optimal trajectory contains a singular arc. We thus have:

$$
\psi(t)=0, \forall t \in\left[t_{1}, t_{2}\right] .
$$

In order to compute the singular control, we derivate two times $\psi$ with respect to $t$. When $\mu(t)$ is given by (7), the system is autonomous on each interval $[0, \bar{T})$ and $(\bar{T}, T]$, and $\psi^{\prime}$ is not necessarily continuous by (19). By derivating two times $\psi$ on each interval, we obtain:

$$
\psi^{\prime \prime}(t)=A(z(t), \lambda(t))+u(t) B(z(t), \lambda(t)),
$$

where $A$ and $B$ are two functions depending on $(z, \lambda)$ (computations are given in the appendix). If the singular arc is optimal, then it satisfies Legendre-Clebsch condition:

$$
B(z(t), \lambda(t)) \geq 0,
$$

see e.g. [10]. Moreover, if $B(z(t), \lambda(t))>0$, then the singular control $u_{s}$ is given by:

$$
u_{s}(t):=-\frac{A(z(t), \lambda(t))}{B(z(t), \lambda(t))} .
$$

When (18) defines a control in $[0,1]$, we say that the singular arc is controllable. When the function $\mu(t)$ is differentiable a.e. (with non-zero derivative), we must take into account the derivative of $\mu$ in the expression of $\psi^{\prime \prime}$. Therefore, $A$ is changed into $\tilde{A}$ where $\tilde{A}$ is given in the Appendix.

\section{NumericAl Results}

\section{A. Direct and indirect methods}

We now solve numerically the problem described in II-B, with both direct and indirect methods.

The direct approach transforms the infinite dimensional optimal control problem into a finite dimensional optimization problem. This is done by a discretization in time applied to the state and control variables, and the dynamics equation.

The indirect approach relies on Pontryagin's Maximum Principle to express the optimal control as a maximizer of a Hamiltonian. This Hamiltonian depends both on the state and the adjoint variables, which are solution of a boundary value problem (BVP). This BVP is still in an infinite dimensional setting, and it can be solved for instance by collocation or shooting methods.

We first try the direct approach with a very simple initialization, namely constant values for the control and state variables over time. Then we analyse the obtained solution to determine the structure of the optimal control, i.e. the number and type of arcs (singular or bang). Moreover, we also extract from this solution some estimates for the switching times, as well as the state and adjoint variables. We recall that the adjoint state for Pontryagin's Principle correspond to the Lagrange multipliers for the dynamics constraints in the discretized problem.

\section{Settings for both methods.}

1. Direct: software BOCOP, discretization by a Lobatto IIIC formula with 1000 time steps, constant initialization, tolerance for IPOPT NLP solver set at $10^{-5}$.

2. Indirect: software SHOOT, ODE solver DOPRI5 with tolerances set at $10^{-12}$, initialization from the direct solution, tolerance for HYBRD solver set at $10^{-14}$.

Computations are done on a Xeon $3.2 \mathrm{GHz}$, and take less than one minute for the direct method, and less than one second for the indirect method.

\section{B. First simulations: discontinuous light model}

Solving the problem with the direct method (Bocop) seems to indicate a control structure of type $\mathbf{B}_{-}-$Singular $-\mathbf{B}_{-}$, with two small arcs $u=0$ at both ends of $[0, T]$. However, the indirect method fails to converge when setting this structure $\mathbf{B}_{-}-$Singular $-\mathbf{B}_{-}$, even with the initialization from the direct method. On the direct solution, we also observe some kind of numerical artifact around $T / 2$, where the light model is discontinuous. By taking more discretization points near $T / 2$, the results seem to indicate the presence of a small bang arc $u=1$. Setting now the structure as $\mathbf{B}_{-}-$Singular $-\mathbf{B}_{+}-$Singular $-\mathbf{B}_{-}$, the indirect method converges easily. We show on Fig. 1, $2,3,4$, the state and control variables over the time, as computed by the direct and indirect methods. Note that the steep change that appears for some variables around $T / 2$ is not a discontinuity, but the result of the short $B_{+}$arc. We observe that both solutions are quite close, as confirmed 
by Table II (note: the actual objective to be minimized is the opposite of the production, hence the minus sign). An interesting fact is that the biomass concentration $(x)$ remains almost constant, with a variation of about only $1 \%$ over the time period. Given the periodicity constraints and the slow dynamics of biological systems, one can expect only small variations of the state variables. Moreover, given the fact that $M:=x+s$ satisfies the equation

$$
\dot{M}=u \beta[\gamma y-M],
$$

$s+x$ will track $\gamma y$. As the substrate concentration $s$ is very small (which is not surprising in order to maximize the conversion of substrate into biogas), this leads to a high biomass concentration $x$, which appears almost constant.

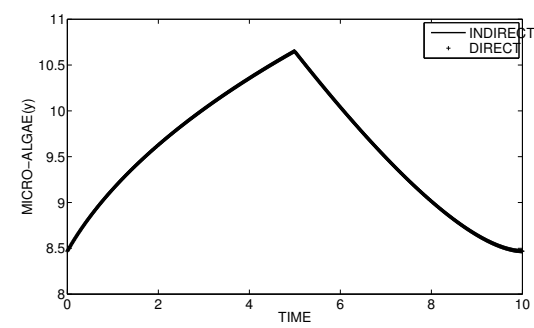

Fig. 1. Micro-algae(y): direct and indirect solutions

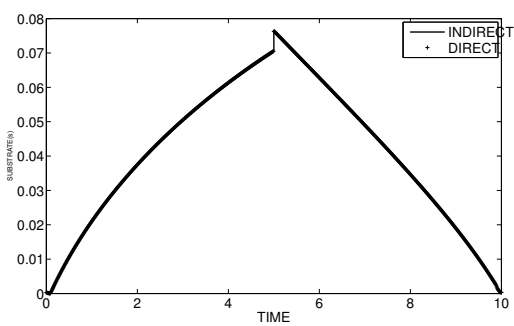

Fig. 2. Substrate(s): direct and indirect solutions

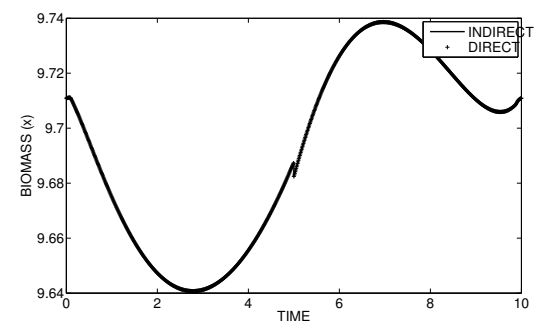

Fig. 3. Biomass(x): direct and indirect solutions

TABLE II

COMPARISON OF THE DIRECT AND INDIRECT METHODS

\begin{tabular}{l|r|r|r|r} 
Method & Objective & $\mathrm{y}(0)$ & $\mathrm{s}(0)$ & $\mathrm{x}(0)$ \\
\hline Direct & -4.03635 & 8.46848 & $2.812 \mathrm{E}-04$ & 9.7109 \\
Indirect & -4.036346 & 8.467771 & $2.829097 \mathrm{E}-04$ & 9.710909
\end{tabular}

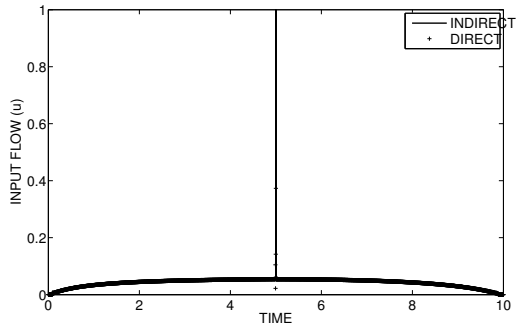

Fig. 4. Input-flow(u): direct and indirect solutions

We also check the control structure and draw the switching function and its first time derivative on Fig. 5. We observe that $\psi$ is negative at the beginning and ending of the time interval, where the control $u=0$. Also, both $\psi$ and $\dot{\psi}$ are equal to zero over the two singular arcs where the control $u \in(0,1)$. Finally, we can see on Fig. 6 the discontinuous jump on $\dot{\psi}$ at $T / 2$ The very small $B_{+}$arc allows to cross this discontinuity while connecting the two singular arcs where $\dot{\psi}=0$. The values for the switching times are: $[0.558778,4.999637,5.000382,9.309402]$, and the length of the $B_{+}$arc is less than $10^{-3}$. This arc is due to the discontinuity of the light model, as confirmed by the study of smoother light functions (IV-C) and the mathematical analysis (IV-D). Also, we note that both $B_{-}$arcs are quite small, and we suspect that they only appear so that the periodicity conditions are satisfied. It would be interesting to investigate theoretically if the periodic constraints (8) can be satisfied by a fully singular trajectory or not.

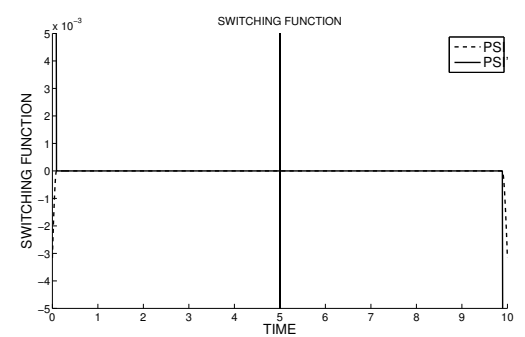

Fig. 5. Switching function and its first derivative

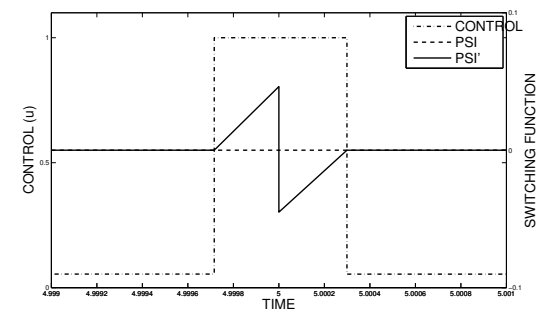

Fig. 6. Switching function: zoom on the light discontinuity

\section{Three light models with different smoothness}

We now compare three light models, discontinuous, continuous and $C^{1}$. The first model is the discontinuous one 
given by 7 , for which we already solved the problem in the previous section. Then we study a continuous model with a linear junction between day and night. Finally, we try a $C^{1}$ model with two quadratic junctions. The three expressions for the function $\mu(\cdot)$ are depicted on Fig. 7 .

The important point is that the smoothness of $\mu$ directly impacts the structure of the optimal control, and more precisely of the singular control. We saw in III-B that the optimal control over a singular arc is the value that equates $\ddot{\psi}$ to $0, \psi$ being the switching function. As $\dot{\psi}$ has the same smoothness as $\mu$, we have three different situations:

i) $\mu$ and $\dot{\psi}$ are discontinuous: the condition $\psi=\dot{\psi}=0$ cannot hold in the general case (see IV-D for more details). Therefore, a singular arc cannot cross the discontinuity. This is why the singular arc is interrupted by a bang arc at the discontinuity at $t=T / 2$, see Fig. 8 .

ii) $\mu$ and $\dot{\psi}$ are continuous (but not $C^{1}$ ): a singular arc can cross the points where $\mu$ is not $C^{1}$, as $\dot{\psi}$ remains equal to 0 . However, $\ddot{\psi}$ is discontinuous where $\mu$ is not $C^{1}$, and we expect the singular control to be discontinuous as well. This is confirmed by the simulations, see the jumps at $t=4$ and $t=6$ on Fig. 9.

iii) $\mu$ is $C^{1}$ and $\ddot{\psi}$ is continuous: the singular control also remains continuous. This is shown on Fig. 10, with junctions times at $t=4,5,6$.

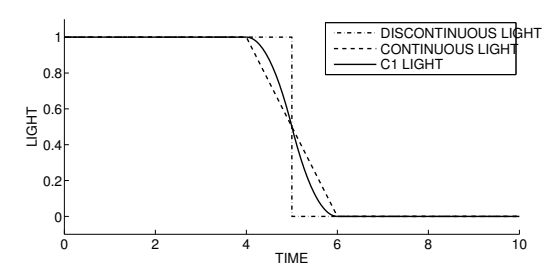

Fig. 7. Three light models: discontinuous, continuous and $C^{1}$

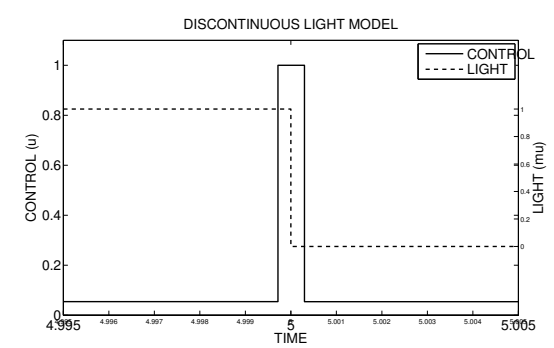

Fig. 8. Discontinuous light model: small bang arc at the discontinuity

Apart from this qualitative differences due to the smoothness of the light model, the three solutions are almost identical, see Table III. In each case, we also check numerically that the Legendre-Clebsch optimality condition is satisifed, namely that $B(z, \lambda)>0$ over the singular arcs (see appendix).

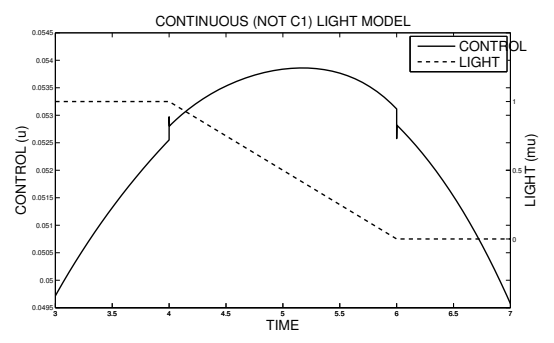

Fig. 9. Continuous light model: singular control is discontinuous

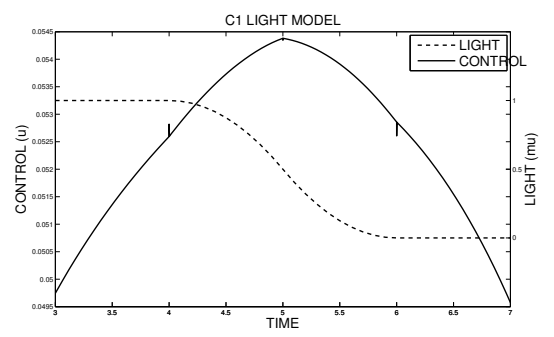

Fig. 10. $C^{1}$ light model: singular control is continuous

\section{Discussion of the optimal solution for the discontinuous light model}

One interesting point which is raised by the numerical computations with the discontinuous light model is to know if the optimal trajectory can be singular in a neighborhood of $t=\bar{T}$. More precisely, we would like to know if this trajectory contains a singular arc on some time interval $\left[t_{1}, t_{2}\right]$, where $0<t_{1}<\bar{T}<t_{2}<T$. In view of the necessary conditions obtained from Pontryagin maximum principle, we believe that this is not possible which explains the presence of an arc $B_{+}$around $t=\bar{T}$.

Indeed, both dynamics (on $[0, \bar{T}]$ and $[\bar{T}, T]$ ) define two different singular curves in $\mathbb{R}^{3}$. If the singular trajectory crosses the junction, then both singular curves should coincide at $\bar{T}$. Now, writing $\phi(\bar{T})=\dot{\phi}\left(\bar{T}^{-}\right)=\dot{\phi}\left(\bar{T}^{+}\right)=0$ gives three linear equations satisfied by the vector $w:=\lambda(\bar{T})$. Together with the conservation of the Hamiltonian (both on $[0, \bar{T}]$ and on $[\bar{T}, T]$ ), we can see that $w$ satisfies a linear system with five equations. From a numerical point of view, we can check that the adjoint vector obtained by Lagrange multiplier does not satisfy these conditions. This explains why the trajectory contains an arc $B_{+}$around $t=\bar{T}$ in order to smooth the possible discontinuity of the trajectory.

We hope that we could answer to this question in a more general setting by analyzing the different necessary conditions on the optimal trajectory at junction. A future work will be addressed to investigate this question more into details.

\section{CONCLUSION}

In this paper we study the coupling of two bioreactors in order to optimize the production of biogas with a culture of microalgae. We analyse the resulting three-dimensional optimal control problem in Lagrange form, and compute the 
TABLE III

COMPARISON OF THE SOLUTIONS FOR THE THREE LIGHT MODELS

\begin{tabular}{l|rrrr} 
Light model & $\mathrm{y}(0)$ & $\mathrm{s}(0)$ & $\mathrm{x}(0)$ & Objective \\
\hline Discontinuous & 8.467771 & $2.829097 \mathrm{E}-04$ & 9.710909 & -4.036346 \\
Continuous & 8.478322 & $4.052398 \mathrm{E}-04$ & 9.699647 & -4.036191 \\
$C^{1}$ & 8.473051 & $3.387794 \mathrm{E}-04$ & 9.705189 & -4.036267
\end{tabular}

value of singular controls. We present a fast and effective numerical strategy, obtaining a first solution by a direct method, and then refining it by an indirect shooting method. We conjecture that optimal trajectoires mostly follow a singular control, concatenated with small bang arcs in order to satisfy both periodic and day-night constraints. Proving the optimality of this strategy, however, remains a difficult theoretical question.

\section{APPENDIX}

The expression of the singular control is obtained by derivating $\psi$ (on each interval $[0, \bar{T}$ ) and $(\bar{T}, T]$ ). For simplicity we write $\mu$ instead of $\mu(t)$. By derivating $\psi$, we get:

$$
\begin{gathered}
\psi^{\prime}(t)=(\gamma y-s)\left(\lambda_{2}-\lambda_{3}\right) \beta x \mu_{2}^{\prime}(s)+\mu_{2}(s) x \\
+y\left[-\lambda_{1} y \frac{\mu}{(1+y)^{2}}+\lambda_{2} \beta \gamma \frac{\mu}{1+y}-r \beta \gamma \lambda_{2}\right]
\end{gathered}
$$

Now, let us define $m_{1}, m_{2}, m_{3}$ by:

$$
\left\{\begin{array}{l}
m_{1}:=(\gamma y-s)\left(\lambda_{2}-\lambda_{3}\right) \beta x \mu_{2}^{\prime}(s) \\
m_{2}:=\mu_{2}(s) x \\
m_{3}:=y\left[-\lambda_{1} y \frac{\mu}{(1+y)^{2}}+\lambda_{2} \beta \gamma\left(\frac{\mu}{1+y}-r\right)\right] .
\end{array}\right.
$$

By derivating, we obtain:

$$
\begin{aligned}
& \dot{m}_{1}=x \beta \mu_{2}^{\prime}(s)(\gamma y-s) u+x \beta\left(\lambda_{2}-\lambda_{3}\right) \times \\
& \quad\left[( \gamma y - s ) \left\{x\left[\mu_{2}^{\prime}(s)^{2}-\mu_{2}(s) \mu_{2}^{\prime \prime}(s)\right]+\mu_{2}^{\prime \prime}(s) \beta(\gamma y-s) u\right.\right. \\
& \left.\left.\quad-\beta \mu_{2}^{\prime}(s) u\right\}+\mu_{2}^{\prime}(s)\left\{\gamma y\left[\frac{\mu}{1+y}-(r+u)\right]+\mu_{2}(s) x\right\}\right]
\end{aligned}
$$

$\dot{m}_{2}=\left(\mu_{2}(s)-u \beta\right) x \mu_{2}(s)+x \mu_{2}^{\prime}(s)\left[-\mu_{2}(s) x+u \beta(\gamma y-s)\right]$.

$$
\begin{aligned}
& \dot{m}_{3}=y\left\{\beta \gamma\left(\frac{\mu}{1+y}-r\right)\left[x\left(\lambda_{2}-\lambda_{3}\right) \mu_{2}^{\prime}(s)+u \lambda_{2} \beta\right]\right\} \\
& +y\left[\frac{\mu}{1+y}-(r+u)\right]\left[-\lambda_{1} y \frac{\mu}{(1+y)^{2}}+\lambda_{2} \beta \gamma\left(\frac{\mu}{1+y}-r\right)\right] \\
& -y\left(\frac{\beta \gamma \mu}{(1+y)^{2}} \lambda_{2}+\frac{\mu(1-y)}{(1+y)^{3}} \lambda_{1}\right)\left[\frac{\mu y}{1+y}-(r+u) y\right] \\
& -y\left\{\frac{y \mu}{(1+y)^{2}}\left(\left[-\frac{\mu}{(1+y)^{2}}+r+u\right] \lambda_{1}-\lambda_{2} \beta \gamma u\right)\right\}
\end{aligned}
$$

Therefore: $\psi^{\prime \prime}(t)=\dot{m}_{1}+\dot{m}_{2}+\dot{m}_{3}$, and we get:

$$
\begin{aligned}
& A(z, \lambda)=x \mu_{2}(s)\left(\mu_{2}(s)-x \mu_{2}^{\prime}(s)\right) \\
& +x \beta\left(\lambda_{2}-\lambda_{3}\right) \times\left[x(\gamma y-s)\left(\mu_{2}^{\prime}(s)^{2}-\mu_{2}(s) \mu_{2}^{\prime \prime}(s)\right)\right] \\
& +x \beta\left(\lambda_{2}-\lambda_{3}\right) \mu_{2}^{\prime}(s)\left(\gamma y\left(\frac{\mu}{1+y}-r\right)+\mu_{2}(s) x\right) \\
& +y\left[\frac{\mu}{1+y}-r\right]\left[-\lambda_{1} y \frac{\mu}{(1+y)^{2}}+\lambda_{2} \beta \gamma\left(\frac{\mu}{1+y}-r\right)\right] \\
& +y \beta \gamma\left(\frac{\mu}{1+y}-r\right) x\left(\lambda_{2}-\lambda_{3}\right) \mu_{2}^{\prime}(s) \\
& -y\left(\frac{\beta \gamma \mu}{(1+y)^{2}} \lambda_{2}+\frac{\mu(1-y)}{(1+y)^{3}} \lambda_{1}\right)\left[\frac{\mu y}{1+y}-r y\right] \\
& -\frac{y^{2} \mu}{(1+y)^{2}}\left[-\frac{\mu}{(1+y)^{2}}+r\right] \lambda_{1},
\end{aligned}
$$

$$
\begin{aligned}
& B(z, \lambda)=x \beta\left[-\mu_{2}(s)+\mu_{2}^{\prime}(s)(\gamma y-s)\right]+x \beta \mu_{2}^{\prime}(s)(\gamma y-s) \\
& +x \beta^{2}\left(\lambda_{2}-\lambda_{3}\right)(\gamma y-s)\left[\mu_{2}^{\prime \prime}(s)(\gamma y-s)-\mu_{2}^{\prime}(s)\right] \\
& -x \beta\left(\lambda_{2}-\lambda_{3}\right) \mu_{2}^{\prime}(s) \gamma y-y\left[-\lambda_{1} y \frac{\mu}{(1+y)^{2}}\right. \\
& \left.+\lambda_{2} \beta \gamma\left(\frac{\mu}{1+y}-r\right)\right]+y \beta^{2} \lambda_{2} \gamma\left[\frac{\mu}{1+y}-r\right] \\
& +y^{2}\left(\frac{\beta \gamma \mu}{(1+y)^{2}} \lambda_{2}+\frac{\mu(1-y)}{(1+y)^{3}} \lambda_{1}\right)-\frac{\mu y^{2}}{(1+y)^{2}}\left(\lambda_{1}-\lambda_{2} \beta \cdot \gamma\right)
\end{aligned}
$$

In the case where $\mu$ is differentiable a.e., we must take into account the derivative of $\mu$ in $m_{3}$. The term $A$ is replaced by $\tilde{A}$ where:

$$
\tilde{A}(z, \lambda):=A(z, \lambda)+y \mu^{\prime}\left[\frac{-\lambda_{1} y}{(1+y)^{2}}+\frac{\lambda_{2} \beta \gamma}{1+y}\right] .
$$

\section{ACKNOWLEDGMENT}

This work was supported by Programa de Financiamiento Basal from the Center of Mathematical Modeling, Universidad de Chile and was developed in the context of DYMECOS INRIA associated team and of the program Stic-AmSud MOMARE. The last author thanks INRIA and the CIRIC (Communication and Information Research and Innovation Center) for providing him a research opportunity.

\section{REFERENCES}

[1] A. Akhmetzhanov, O. Bernard, F. Grognard and P. Masci, Optimization of a photobioreactor biomass production using natural light, In Proceedings of the 49th CDC conference, 2010.

[2] M.J. Barbosa and R.H. Wijffels, An Outlook on Microalgal Biofuels, Science, vol. 329, pp. 796-799, 2010.

[3] G. Bastin and D. Dochain, On-line estimation and adaptive control of bioreactors, Elsevier, New York, 1990.

[4] O. Bernard, N. Bernet and B. Sialve, Anaerobic digestion of microalgae as a necessary step to make microalgal biodiesel sustainable, Biotechnology Advances, vol. 27, pp. 409-416, 2009.

[5] J.-F. Bonnans, V. Grelard, P. Martinon, Bocop, the optimal control solver, Open source toolbox for optimal control problems, http: / /bocop.org, 2011.

[6] D. Dochain, A. Rapaport, Minimal time control of fed-batch processes for growth functions with several maxima, IEEE Transactions on Automatic Control, vol. 56, 11, pp. 2671-2676, 2011.

[7] L. Cesari, Optimization - Theory and Applications, Problems with Ordinary Differential Equations, Springer-Verlag, New-York, 1983.

[8] E. Duran, A. Isambert, C. Joannis-Cassan and P. Spolaore, Commercial applications of microalgae, Journal of bioscience and bioengineering, vol. 101, pp. 87-96, 2006. 
[9] A.N. Kolmogorov, Sulla teoria di Volterra della lotta per l'esistenza, Giornale Istituto Ital. Attuari, vol. 7, pp. 74-80, 1936.

[10] H.M. Robbins, A Generalized Legendre-Clebsch Condition for the Singular Cases of Optimal Control. IBM J. of Research and Development 11, 361-372, 1967.

[11] H.L. Smith and P. Waltman, The theory of the chemostat, Dynamics of microbial competition, Cambridge University Press, 1995.

[12] G. Wolkowicz and X.Q. Zhao, N-Species Competition in a Periodic Chemostat, Differential Integral Equations, vol. 11, no. 3, 465-491, 1998.

[13] X.Q. Zhao, Dynamical systems in population biology, Springer-Verlag, 2003.

[14] F. Zanolin, Continuation theorems for the periodic problem via the translation operator, Rend. Sem. Mat. Univ. Pol. Torino, vol. 54, 1, 1996. 